\title{
Minería y glaciares rocosos: impactos ambientales, antecedentes políticos y legales, y perspectivas futuras ${ }^{1}$
}

\author{
Alexander Brenning² y Guillermo F. Azócar ${ }^{3}$
}

\begin{abstract}
RESUMEN
El interés científico y político en los glaciares rocosos andinos ha aumentado fuertemente en los últimos años debido al creciente reconocimiento de su importancia hidrológica y las recientes intervenciones mineras en ellos. Se presenta un resumen de los conocimientos científicos y el marco político-legal de las intervenciones pasadas y actuales en glaciares rocosos en Chile y, en forma preliminar, en Argentina y Perú. En estos países, 26 faenas mineras han afectado glaciares rocosos o podrían posiblemente intervenirlos en el futuro. Hasta la fecha, el área afectada de glaciares rocosos en Chile es de aproximadamente $3,3 \mathrm{~km}^{2}$. Se recomienda ampliar el actual debate sobre intervenciones en glaciares rocosos, basado en la actualidad principalmente en argumentos hidrológicos, para tomar en cuenta peligros ambientales en el largo plazo -como la posible inestabilidad de glaciares rocosos con depósitos mineros- como también la amplia gama de servicios ambientales brindados por los glaciares rocosos.
\end{abstract}

Palabras clave: Glaciar rocoso, minería, recursos hídricos, estabilidad de ladera, servicios ambientales.

\begin{abstract}
The scientific and political interest in Andean rock glaciers has strongly grown in recent years due to the increasing recognition of their hydrological importance and recent impacts of mining on rock glaciers. We present a review of the scientific knowledge and political and legal context of past and current impacts on rock glaciers in Chile and, in a preliminary form, in Argentina and Peru. In these countries, 26 mining projects have already affected rock glaciers or may possibly do so in the future. Until now, the affected rock glacier area in Chile is approximately 3.3 square kilometres. We recommend broadening the current discussion about interventions of rock glaciers, which is mainly based on hydrological arguments, to take into account both long-term environmental hazards such as the possible instability of rock glaciers with waste rock dumps, and the broad set of environmental services provided by rock glaciers.
\end{abstract}

Key words: Rock glacier, mining, water resources, slope stability, environmental services.

1 Artículo recibido el 9 de noviembre de 2009 y aceptado el 4 de enero de 2010.

2 Department of Geography and Environmental Management, University of Waterloo (Canadá). E-mail: brenning@uwaterloo.ca
3 Unidad Geografía Proyectos UC, Pontificia Universidad Católica de Chile (Chile). E-mail: gmoazocar@ gmail.com 
Las fuertes intervenciones mineras sobre glaciares rocosos en Chile son hasta ahora hechos únicos a nivel mundial (Brenning, 2008), y están en directa relación con el desarrollo minero experimentado en las últimas tres décadas. Los impactos ambientales sobre estos cuerpos de hielo en la cordillera de los Andes solo últimamente han recibido la atención científica y de los medios de comunicación. Recientes estudios realizados por Brenning $(2005,2008)$ y Azócar y Brenning (2008) describen por primera vez la remoción y degradación de glaciares rocosos por faenas mineras en Chile central, y evalúan su importancia como recurso hídrico en esta zona semiárida. En este contexto el presente trabajo tiene por objetivo revisar, ampliar y sistematizar el conocimiento actual de las intervenciones sobre glaciares rocosos en los Andes chilenos en su contexto científico y políticolegal. Ello no solo es de importancia regional a nivel de los países andinos (Bórquez et al., 2006; CONAMA, 2009), sino también a nivel internacional en el contexto de intervenciones en la criósfera (Hasslacher, 2005; Bütler, 2007; Kronenberg, 2009) y la actividad minera en zonas montañosas en general (Szwilski et al., 2001; Palmer et al., 2010).

\section{Estructura y métodos}

El presente trabajo integra aspectos geomorfológicos y ambientales con antecedentes político-legales con el fin de presentar por primera vez una visión transversal del problema de las intervenciones mineras y la protección de glaciares rocosos.

En la primera parte del artículo se parte por resumir los conocimientos geográficos sobre los glaciares rocosos andinos revisando la literatura geomorfológica y se crea una tipología de las intervenciones en glaciares rocosos según su magnitud y los potenciales efectos ambientales. En la segunda parte del trabajo se analiza el marco político-legal de la protección de glaciares rocosos, incluyendo la Política Nacional de Glaciares de Chile. Con este fin se evalúan textos gubernamentales y legales respecto de su relevancia para la protección de glaciares rocosos.

La tercera parte del artículo utiliza principalmente métodos geomorfológicos, en particular la interpretación de fotografías aéreas e imágenes satelitales y de informes técnicos, para reconstruir la historia ambiental de intervenciones pasadas en glaciares rocosos e identificar áreas con posibles intervenciones futuras. Aunque el mayor énfasis de este análisis está en la situación chilena, también se presentan primeros resultados para Argentina y Perú. Como fuentes de información se usaron: 1) los estudios o declaraciones de impacto ambiental de los proyectos y las resoluciones correspondientes; 2) publicaciones, noticias e informes técnicos relacionados con los proyectos mineros; 3) la interpretación de fotografías aéreas de los vuelos Geotec, años 1996-2002 del Servicio Aerofotogramétrico de la Fuerza Aérea de Chile, y Hycon, 1955/56 del Instituto Geográfico Militar; y 4) la interpretación de imágenes satelitales disponibles en Google Earth. Con el fin de estimar, en forma conservadora, el equivalente en agua de los glaciares rocosos afectados, asumimos que la capa rica en hielo del permafrost de un glaciar rocoso tiene un espesor de $20 \mathrm{~m}$, un contenido volumétrico promedio de hielo entre $40 \%-60 \%$, y una densidad del hielo de 0,9 g/cm³ (Brenning, 2005; Azócar \& Brenning, 2010).

\section{Fundamentos científicos}

\section{Los glaciares rocosos}

Los glaciares rocosos (también llamados glaciares de roca o de escombros) son la expresión geomorfológica de la reptación de permafrost de montaña con alto contenido de hielo (Barsch, 1996; Burger et al., 1999; Haeberli, 2000). Poseen una morfología en forma de lengua o lóbulo y un escarpe frontal y lateral como resultados del desplazamiento lento ladera o valle abajo, presentando cordones y surcos en su superficie (Figura $N^{0} 1$ ). La estructura interna de los glaciares rocosos está constituida por una mezcla de hielo (entre $40 \%$ y $60 \%$ ) y material detrítico (Barsch, 1996; Hoelzle et al., 1998; Arenson et al., 2002), convirtiéndolos en reservorios de agua congelada acumulada durante el período postglacial. Las tasas de desplazamiento horizontal generalmente varían entre los $10 \mathrm{~cm}$ y $100 \mathrm{~cm}$ por año (Burger et al., 1999; Roer et al., 2005). Su longitud va desde unos pocos metros a varios kilómetros pero típicamente varía entre 200 m y 800 m. Los glaciares rocosos se originan principalmente a partir 
de material detrítico gravitacional y/o morrénico. Según su dinámica se clasifican entre formas activas (en movimiento y con hielo en su interior), inactivas (sin movimiento pero aún con hielo en su interior) y fósiles (sin movimiento y donde se ha fusionado completamente su contenido de hielo) (Barsch, 1996).

Figura $\mathrm{N}^{0} 1$

Glaciar rocoso activo en la cuenca del río Huasco, Región de Copiapó

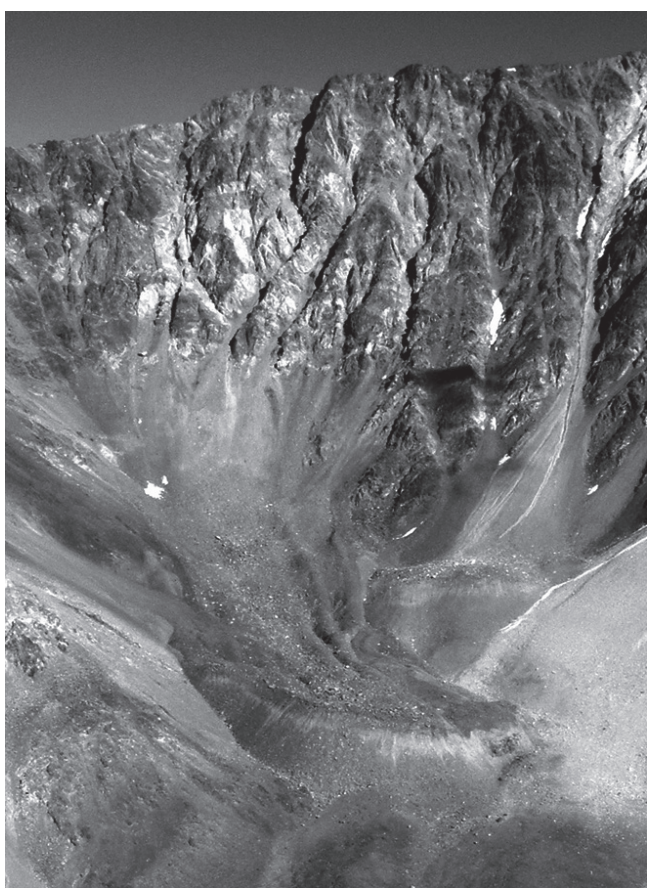

Fuente: Colección personal de los autores.

\section{Rol ambiental}

La importancia relativa de los glaciares rocosos como reservorios de agua varía entre las diferentes zonas de alta montaña del mundo, según la abundancia de estas formas y la magnitud de la glaciación actual. En regiones montañosas áridas y semiáridas de la cordillera de los Andes, especialmente en sectores cordilleranos del norte y centro de Chile y Argentina, la importancia como reservorios de agua naturales se acrecienta debido a la presencia de una gran cantidad de glaciares rocosos de diversas formas y tamaños (Trombotto et al., 1999; Azócar \& Brenning, 2010). Los glaciares rocosos pueden almacenar más agua que los glaciares "blancos", como es el caso en Chile entre los $29^{\circ}$ a $32^{\circ} \mathrm{S}$ (Azócar \& Brenning, 2010).

Los servicios ambientales de los glaciares rocosos son similares a aquellos de los glaciares blancos (Kronenberg, 2009). Entre los servicios relacionados a su valor de uso, están principalmente su uso como fuente de agua, su contribución a la regulación del clima, su contenido de información sobre el pasado geológico y climático (Barsch, 1996; Brenning, 2005), y su apreciación en el turismo (Parks Canada, 2008). A ello se agrega el valor no relacionado al uso, como el de su existencia, de legado y altruista (Kronenberg, 2009). Por otro lado, en el aspecto económico los glaciares rocosos también pueden tener un valor negativo al crear una barrera al desarrollo de un proyecto o al causar daño por su movimiento o colapso (Grebenets et al., 1997; Burger et al., 1999; Valenzuela, 2004; Kääb et al., 2005; Kääb et al., 2007).

\section{Tipología de intervenciones y sus efectos ambientales}

En este trabajo se distinguen tres principales tipos de intervenciones en glaciares rocosos (Cuadro $\mathrm{N}^{\circ} 1$ ): 1) remoción completa o parcial; 2) construcción de depósitos de lastre (roca estéril de baja ley) sobre glaciares rocosos; y 3) construcción de infraestructura, principalmente caminos sobre ellos (Brenning, 2008). El principal efecto de la remoción de glaciares rocosos está en la pérdida irreversible de un recurso hídrico no renovable. También se elimina del sistema hidrológico un componente que contribuye a los caudales de los ríos durante los meses de verano y particularmente en años calurosos (Burger et al., 1999; Azócar \& Brenning, 2010), y se modifica el paisaje. El caso emblemático de este tipo de intervención se da en Codelco División Andina (Brenning, 2008).

La depositación de lastre sobre glaciares rocosos tiene como consecuencia cambios en su dinámica y por lo tanto en la estabilidad de la forma, tanto en el corto como en el largo plazo. Tales efectos ya han sido observados en la minería chilena, como se verá 
más adelante. La posible degradación del permafrost de los glaciares rocosos por efectos geotérmicos y geoquímicos (Acid Rock Drainage) y la agradación de permafrost con variables contenidos de hielo en el material depositado tienen incalculables consecuencias en el largo plazo (décadas a siglos) para la estabilidad de la forma (Grebenets et al., 1997; Hollesen et al., 2009). El colapso de un glaciar rocoso recubierto con depósito de lastre no solo causaría daños en las cercanías, sino también debe considerarse la posibilidad de que la mezcla de detritos, hielo, y material de arrastre -en particular, agua y lodos de estanques de relave- se convierta en un flujo detrítico capaz de desplazarse largas distancias (Valenzuela, 2004; Brenning, 2008). El derrumbe y flujo detrítico del río Colorado en 1987 (Casassa \& Marangunic, 1993) es un ejemplo natural de este tipo de movimiento en masa que puede afectar a asentamientos humanos localizados a gran distancia del origen del derrumbe.

La construcción de caminos y otros tipos de infraestructura básica en glaciares rocosos puede afectar en forma local el balance energético del permafrost a través de la compactación y remoción vertical y horizontal del material, resultando en una posible degradación del permafrost. Del mismo modo debe considerarse el daño que puede producir el movimiento del glaciar rocoso sobre esta infraestructura, por lo que se recomienda evitar los glaciares rocosos para todo tipo de construcción (Burger et al., 1999). De la misma manera, el riesgo de accidentes y contaminación debido al desplazamiento de vehículos

\section{Cuadro $\mathrm{N}^{\circ} 1$}

Tipología de intervenciones mineras en glaciares rocosos y sus posibles consecuencias ambientales

\begin{tabular}{|c|c|c|c|}
\hline $\begin{array}{c}\text { Tipo de } \\
\text { intervención }\end{array}$ & Posibles consecuencias ambientales & $\begin{array}{l}\text { Posibles consecuencias } \\
\text { económicas }\end{array}$ & $\begin{array}{l}\text { Efectos en } \\
\text { el tiempo }\end{array}$ \\
\hline \multirow{2}{*}{$\begin{array}{l}\text { Remoción } \\
\text { completa o } \\
\text { parcial de } \\
\text { glaciares rocosos }\end{array}$} & $\begin{array}{l}\text { Pérdida de un reservorio de hielo subterráneo } \\
\text { fósil, reducción de caudales de ríos }\end{array}$ & $\begin{array}{l}\text { Disponibilidad reducida de } \\
\text { agua en la cuenca }\end{array}$ & $\begin{array}{l}\text { Cambio } \\
\text { inmediato }\end{array}$ \\
\hline & Alteración irreversible del paisaje & $\begin{array}{l}\text { Reducción del valor turístico } \\
\text { del paisaje }\end{array}$ & $\begin{array}{l}\text { Cambio } \\
\text { inmediato }\end{array}$ \\
\hline \multirow[t]{4}{*}{$\begin{array}{l}\text { Creación de } \\
\text { depósitos de } \\
\text { lastre sobre } \\
\text { glaciares rocosos }\end{array}$} & $\begin{array}{l}\text { Aceleramiento, inestabilidad y posible } \\
\text { colapso de glaciares rocosos }\end{array}$ & $\begin{array}{l}\text { Riesgo para vidas humanas } \\
\text { e infraestructura minera } \\
\text { y pública; necesidad de } \\
\text { monitoreo y control }\end{array}$ & $\begin{array}{l}\text { Meses a } \\
\text { décadas }\end{array}$ \\
\hline & $\begin{array}{l}\text { Contaminación de agua almacenada en } \\
\text { glaciares rocosos como consecuencia del } \\
\text { Acid Rock Drainage (ARD) }\end{array}$ & $\begin{array}{l}\text { Necesidad de monitoreo y } \\
\text { tratamiento del agua; mayor } \\
\text { escasez de agua }\end{array}$ & $\begin{array}{l}\text { Años a } \\
\text { décadas }\end{array}$ \\
\hline & $\begin{array}{l}\text { Degradación del permafrost y posible } \\
\text { inestabilidad de glaciares rocosos como } \\
\text { consecuencia de la producción de calor en } \\
\text { reacciones exotérmicas del ARD }\end{array}$ & $\begin{array}{l}\text { Riesgo para vidas humanas } \\
\text { e infraestructura minera y } \\
\text { pública }\end{array}$ & $\begin{array}{l}\text { Años a } \\
\text { décadas }\end{array}$ \\
\hline & $\begin{array}{l}\text { Degradación basal del permafrost de } \\
\text { glaciares rocosos por efecto del gradiente } \\
\text { térmico; posible inestabilidad basal }\end{array}$ & $\begin{array}{l}\text { Riesgo para vidas humanas } \\
\text { e infraestructura minera y } \\
\text { pública }\end{array}$ & $\begin{array}{l}\text { Décadas a } \\
\text { siglos }\end{array}$ \\
\hline \multirow[t]{2}{*}{$\begin{array}{l}\text { Construcción de } \\
\text { caminos sobre } \\
\text { glaciares rocosos }\end{array}$} & $\begin{array}{l}\text { Degradación superficial del permafrost por la } \\
\text { alteración de propiedades físicas superficiales } \\
\text { como el albedo y la conductividad térmica }\end{array}$ & $\begin{array}{l}\text { Peligro para usuarios del } \\
\text { camino }\end{array}$ & $\begin{array}{l}\text { Años a } \\
\text { décadas }\end{array}$ \\
\hline & $\begin{array}{l}\text { En caso de accidentes, contaminación con } \\
\text { sustancias tóxicas transportadas (por ejemplo } \\
\text { combustible) }\end{array}$ & $\begin{array}{l}\text { Disponibilidad reducida de } \\
\text { agua en la cuenca; necesidad } \\
\text { de monitoreo }\end{array}$ & $\begin{array}{l}\text { Meses a } \\
\text { años }\end{array}$ \\
\hline
\end{tabular}

Fuente: Elaboración propia. 
sobre una superficie inestable y el posible derrame de sustancias peligrosas (por ejemplo combustibles) están presentes, sin embargo, este riesgo dependerá principalmente de la frecuencia del uso del camino, el tipo de carga, entre otros.

\section{Antecedentes político-legales}

La preocupación política y legal sobre la protección y conservación de los glaciares blancos y rocosos en Chile es de carácter reciente, a consecuencia de la actual discusión sobre los efectos del cambio climático global y el impacto público del proyecto minero Pascua-Lama (Kronenberg, 2009). A continuación se revisan los principales cuerpos legales y normativas políticas que regulan la protección y conservación de glaciares en Chile, parcialmente basados en Bórquez et al. (2006).

El Código de Aguas es el principal reglamento legal para la gestión del uso del agua en Chile. En general norma las aguas líquidas y no menciona a los glaciares blancos y rocosos dentro de sus definiciones. A pesar de que los glaciares rocosos constituyen aguas terrestres subterráneas que por su flujo formarían parte de la "corriente" correspondiente a su cuenca hidrográfica (Código de Aguas, Artículos 1-3), se sostiene que el Código norma solo las aguas líquidas y no incluye las sólidas (Bórquez et al., 2006). También es posible que la inclusión de los glaciares rocosos en el Código de Aguas facilitara su uso (Bórquez et al., 2006).

Por otra parte, a través de las regulaciones del Sistema Nacional de Áreas Silvestres Protegidas por el Estado (SNASPE; Ley No 18.362 de 1984) y la de Santuarios de la Naturaleza (Ley $\mathrm{N}^{\circ} 17.288$ de Monumentos Nacionales, 1970), se da una cierta protección a los glaciares blancos y glaciares rocosos localizados al interior de las áreas protegidas al prohibir o limitar el uso con fines económicos en ellas, dependiendo del tipo de área protegida. Sin embargo, solo un pequeño número de glaciares rocosos en Chile está protegido de esta forma, y está permitida la realización de actividades de prospección minera al interior de estas áreas (Ley 18.248, Código de Minería).

Por otro lado, legislaciones ambientales tales como la Ley 19.300 sobre Bases Ge- nerales del Medio Ambiente del año 1994 y el Reglamento del Sistema de Evaluación de Impacto Ambiental (SEIA) de 1997 son los principales cuerpos legales que han regulado muchas de las actividades susceptibles de causar impactos en áreas con presencia de glaciares. A partir de una reciente modificación (Artículo No 3 D. S. No 95 de 2001, modificado el 29/11/2008), propuesta como parte de las líneas estratégicas de la política para la protección y conservación de glaciares (o Política Nacional de Glaciares, PNG) se establece que todo proyecto susceptible de causar impacto ambiental sobre un glaciar deberá someterse al Sistema de Evaluación de Impacto Ambiental (SEIA). No obstante, una importante limitación de esta modificación es que solo considera dentro de su ámbito a los glaciares que se encuentran incorporados en un inventario público de la Dirección General de Aguas (DGA), los que son incompletos en su forma actual.

Debido al estancamiento en las iniciativas legales sobre la protección de glaciares en Chile a raíz de falta de consenso al interior del Ejecutivo como asimismo por presiones del sector minero (Business News Americas, 2007; El Mostrador, 2008), se desarrolla durante el año 2008 la PNG, iniciativa que tiene por objetivo preservar y conservar los glaciares de Chile. Entre las medidas concretas mencionadas están las modificaciones al SEIA, la construcción de un nuevo registro de glaciares y el aumento en la investigación científica sobre el tema (CONAMA, 2009). Para efectos de esta Política, se entiende por glaciar "toda masa de hielo perenne, formada por acumulación de nieve, cualquiera que sean sus dimensiones y sus formas", y se incluyen explícitamente los glaciares rocosos en esta definición al interpretarlos como "glaciares recubiertos". Según la definición citada, pequeños campos de nieve perenne que son muy frecuentes en zonas de alta montaña también se considerarían como glaciares, contrario a las definiciones establecidas en la literatura científica, la que recomienda exigir un tamaño mínimo de $0,1 \mathrm{~km}^{2}$ para que se trate de un glaciar (Haeberli, 2000); los glaciares rocosos, sin embargo, pueden ser más pequeños (Barsch, 1996). Además de establecer medidas para su preservación y conservación, la PNG también contempla "eventuales intervenciones, si los intereses superiores de 
la Nación así lo exigen", no obstante, la PNC no define los criterios necesarios para evaluar esta condición.

Un detalle notable de la modificación reciente al SEIA es que los glaciares se consideran como recursos naturales renovables (Artículo 6). Ello es claramente opuesto a las definiciones científicas de los recursos renovables, según las que estos tienen un tiempo de regeneración de años hasta algunas décadas (Cuff, 2008). Los glaciares en retroceso y los glaciares rocosos, que se han formado en miles de años, son recursos cuasi renovables (tiempo de regeneración de siglos a milenios), iguales que, por ejemplo, los suelos y el agua subterránea. El manejo sustentable de un recurso cuasi renovable sin duda difiere en forma fundamental del manejo sustentable de un recurso renovable.

En forma paralela en Argentina se han desarrollado iniciativas en la materia. Durante el año 2007 se presentó un proyecto de ley llamado de Presupuestos mínimos para la Protección de los Glaciares y del Ambiente Periglacial (Ley $N^{0}$ 26.418) que fue aprobado el año 2008 por la Cámara de Diputados. Sin embargo, fue vetada ese mismo año por la presidenta argentina debido a que la ley incluye una prohibición absoluta de ciertas actividades en ambientes periglaciales saturados en hielo (léase glaciar rocoso; Decreto $\left.N^{\circ} 1837 / 2008\right)$. Finalmente durante octubre de 2010 fue aprobada una nueva versión de la ley, con cambios en el alcance de las definiciones de glaciar y ambiente periglacial. Esta nueva ley prohíbe intervenciones en glaciares y glaciares rocosos, y establece nuevas exigencias a las explotaciones mineras en alta montaña. Además, considera a los glaciares como bienes de carácter público.

\section{Intervenciones en glaciares rocosos en Chile}

Las intervenciones mineras sobre glaciares rocosos en los Andes de Chile en su mayoría han ocurrido en las últimas décadas y se extienden desde los $26^{\circ} \mathrm{S}$ a los $34^{\circ} \mathrm{S}$, no obstante la mayor parte de las intervenciones se han concentrado a los $33^{\circ} \mathrm{S}$ en las regiones de Valparaíso y Metropolitana. La Figura $N^{\circ} 2$ muestra la distribución espacial de las faenas mineras que han perturbado o potencialmente afectarán glaciares rocosos en el futuro, tanto en los Andes chilenos como argentinos. Esta sección presenta en forma detallada cada una de las intervenciones sobre glaciares rocosos detectadas en Chile, y la sección siguiente da un resumen de proyectos con potenciales impactos futuros en Chile, Argentina y Perú.

\section{Los Bronces, Región Metropolitana}

Los Bronces $\left(33^{\circ} 9^{\prime} \mathrm{S}, 70^{\circ} 16^{\prime} \mathrm{W}\right)$ está situado a los 3.500 m.s.n.m. en la cuenca del río Mapocho y a $40 \mathrm{~km}$ de la ciudad de Santiago. Descubierta en el año 1862, la explotación del mineral se inició en 1906. Durante el siglo XX tuvo propietarios tanto del sector público (ENAMI) como del privado (Exxon Minerals), y finalmente fue adquirida el año 2002 por Anglo American Chile. La producción anual es de 232 kt de cobre (Editec, 2006).

Los glaciares rocosos en el área de Los Bronces y la vecina División Andina de Codelco (Región de Valparaíso) fueron los primeros que se han estudiado en Chile (Lliboutry, 1961). Los impactos sobre glaciares rocosos han sido ampliamente documentados en el área, especialmente aquellos generados por el depósito de lastre sobre el glaciar rocoso Infiernillo (Contreras e Illanes, 1992; Valenzuela, 2004; Brenning, 2008). De los $1,9 \mathrm{~km}^{2}$ de glaciares rocosos en Los Bronces, se estima que hasta el 1997 un área de 0,2 $\mathrm{km}^{2}$ de glaciares rocosos fue afectada -presumiblemente removida- como consecuencia del crecimiento del rajo de la mina; otros 0,2 $\mathrm{km}^{2}$ fueron cubiertos por el depósito de lastre Infiernillo (descrito más adelante con más detalle), y $0,4 \mathrm{~km}^{2}$ son afectados por la construcción de infraestructura, principalmente caminos (Brenning, 2008). El equivalente en agua afectada hasta 1997 es entre los 6 millones de $\mathrm{m}^{3}$ y 9 millones de $\mathrm{m}^{3}$ (Cuadro $\mathrm{N}^{\circ} 2$ ).

\section{Codelco División Andina, Región de Valparaíso}

Codelco División Andina $\left(33^{\circ} 9^{\prime} \mathrm{S}, 70^{\circ}\right.$ $\left.15^{\prime} \mathrm{W}\right)$, vecina a Los Bronces, es el complejo minero que ha causado las mayores intervenciones en glaciares rocosos a nivel mundial, y que potencialmente afectará más glaciares en el futuro si se desarrolla el yacimiento El 
Americano (Área Minera, 2009). Comenzó su operación a gran escala en los años 1980 a través de explotaciones a rajo abierto y subterráneas, y hasta el año 2007 aumentó su producción de cobre a 218 kt (Codelco, 2008).
Las operaciones de División Andina han tenido impactos en glaciares rocosos por lo menos desde que la mina a rajo abierto, Sur Sur, comenzó a operar en 1980. Desde entonces, dos glaciares rocosos (área total

Figura $\mathrm{N}^{\circ} 2$

Faenas mineras en zonas altoandinas de Chile y Argentina*

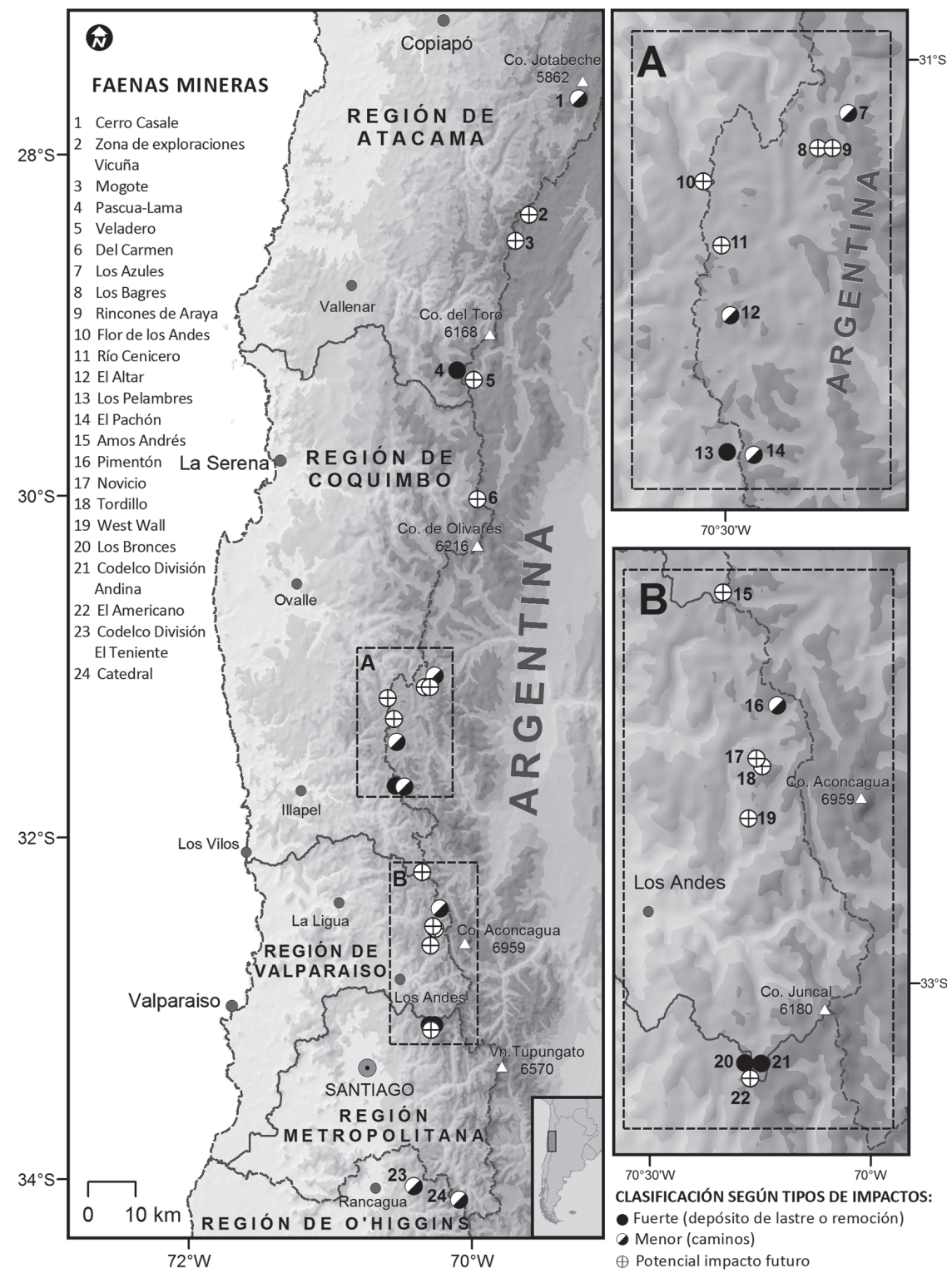

*El proyecto Agua Rica en la sierra del Aconquija (Argentina) está fuera del área del mapa. Fuente: Elaboración propia. 
Cuadro $\mathrm{N}^{\circ} 2$

Área intervenida y equivalente en agua afectada según proyecto minero en Chile

\begin{tabular}{|c|c|c|c|c|c|c|c|}
\hline \multirow[t]{2}{*}{ Faena minera } & \multirow{2}{*}{$\begin{array}{l}\text { Área original } \\
\text { de glaciares } \\
\text { rocosos } \\
\left(\mathrm{km}^{2}\right)\end{array}$} & \multicolumn{2}{|c|}{$\begin{array}{l}\text { Área intervenida (o por } \\
\text { intervenir) de glaciares } \\
\text { rocosos } \\
\left(\mathrm{km}^{2}\right)\end{array}$} & \multicolumn{2}{|c|}{$\begin{array}{l}\text { Equivalente en agua } \\
\text { de glaciares rocosos } \\
\text { intervenidos (o por } \\
\text { intervenir) }\left(10^{6} \mathrm{~m}^{3}\right)\end{array}$} & \multirow{2}{*}{$\begin{array}{c}\text { Años de } \\
\text { inicio de la } \\
\text { intervención }\end{array}$} & \multirow{2}{*}{$\begin{array}{l}\text { Declarado } \\
\text { en EIA o } \\
\text { DIA }^{\mathrm{a}}\end{array}$} \\
\hline & & $\begin{array}{l}\text { Remoción, } \\
\text { depósito de } \\
\text { lastre }\end{array}$ & $\begin{array}{c}\text { Caminos, } \\
\text { sondajes }\end{array}$ & $\begin{array}{l}\text { Remoción, } \\
\text { depósito de } \\
\text { lastre }\end{array}$ & $\begin{array}{l}\text { Caminos, } \\
\text { sondajes }\end{array}$ & & \\
\hline $\begin{array}{l}\text { Codelco } \\
\text { División } \\
\text { Andina }\end{array}$ & 2,6 & 1,32 & $0,78^{b}$ & $10-14$ & $6-8$ & $\begin{array}{l}\text { Desde antes } \\
\text { de } 1990\end{array}$ & $\begin{array}{l}\text { Sí (EIA } \\
2001)\end{array}$ \\
\hline Los Bronces & 1,9 & 0,4 & 0,4 & $3-4$ & $3-4$ & $\begin{array}{l}\text { Desde antes } \\
\text { de } 1990\end{array}$ & No \\
\hline Los Pelambres & 0,4 & 0,1 & 0,1 & $0,8-1,3$ & $1-1,6$ & 2003-2004 & No \\
\hline Pascua-Lama & 0,3 & 0,03 & - & $0,2-0,4$ & - & $\begin{array}{l}\text { A partir de } \\
2013\end{array}$ & $\begin{array}{l}\text { Sí (EIA } \\
\text { 2004-2006) }\end{array}$ \\
\hline Pimentón & $>1,6$ & - & 0,06 & - & $0,4-0,6$ & $\begin{array}{l}\text { Desde antes } \\
\text { de } 1996\end{array}$ & No \\
\hline $\begin{array}{l}\text { Codelco } \\
\text { División El } \\
\text { Teniente }\end{array}$ & n.d. & - & 0,04 & - & $0,3-0,4$ & $\begin{array}{l}\text { Antes de } \\
1997\end{array}$ & No \\
\hline Catedral & 2,3 & - & 0,03 & - & $0,2-0,3$ & $\begin{array}{l}\text { Entre } 1996 \\
\text { y } 2001\end{array}$ & No \\
\hline Cerro Casale & 0,1 & - & 0,01 & - & 0,1 & $\begin{array}{l}\text { Desde antes } \\
\text { de } 1999\end{array}$ & No \\
\hline
\end{tabular}

a "No": proyecto no tiene DIA/EIA, intervenciones se produjeron antes de la introducción del SEIA, o proyecto tiene DIA/EIA pero no anuncia intervenciones en glaciares rocosos.

b Incluye áreas de depósitos de lastre sobre glaciares rocosos.

n.d.: no determinado

Fuente: Elaboración propia.

$\left.1,32 \mathrm{~km}^{2}\right)$ identificados por Lliboutry (1961) han desaparecido casi completamente, y dos depósitos de lastre han cubierto glaciares rocosos (Brenning, 2008; Cuadro No 2). Un ejemplo de estas intervenciones se muestra en la Figura $\mathrm{N}^{\circ} 3$. Adicionalmente $0,78 \mathrm{~km}^{2}$ de glaciares rocosos han sido intervenidos a través de la construcción de depósitos de lastre o de infraestructura minera (Brenning, 2008). Los problemas de estabilidad relacionados a estos depósitos se presentan en la sección siguiente. En total, el equivalente en agua afectada se calcula entre 15 millones de $\mathrm{m}^{3}$ y 23 millones de $\mathrm{m}^{3}$.

Codelco admite haber removido glaciares rocosos (Minergía, 2006a). Intervenciones efectuadas después de 2001 fueron anuncia- das en el EIA de un proyecto de expansión y aprobadas por CONAMA (Brenning, 2008). Según las informaciones disponibles, las remociones anteriores, efectuadas principalmente a partir de 1999 (Morales-Carvajal, 2001) y por lo tanto posteriores al establecimiento del SEIA, no contaban con tal autorización.

Codelco recientemente anunció el descubrimiento de una yacimiento de gran tamaño llamado El Americano (Área Minera, 2009). Este se encuentra al sur de la actual mina, principalmente en la zona del actual Santuario de la Naturaleza Yerba Loca, a gran profundidad y debajo de glaciares (Área Minera, 2009), en una zona con un gran número de glaciares rocosos. El futuro desarrollo de esta 
reserva permitirá evaluar la efectividad de la Ley de Monumentos Nacionales en la protección de glaciares rocosos en Chile.

\section{Problemas de estabilidad en depósitos de lastre en Los Bronces y División Andina}

La construcción de depósitos de lastre sobre glaciares rocosos desde la década del 1980 en Los Bronces y División Andina ha causado serios problemas de estabilidad (Apablaza et al., 2001; Valenzuela, 2004). El caso del glaciar rocoso Infiernillo de Los Bronces es el ejemplo mejor documentado de tal depósito. Este glaciar rocoso tiene una superficie de $1,0 \mathrm{~km}^{2}$ y un largo de $2,5 \mathrm{~km}$, formando una impresionante lengua que sale de un circo glacial ubicado sobre la mina Los Bronces (Brenning, 2008). Teniendo un espesor promedio de $35 \mathrm{~m}$ (Valenzuela, 2004), su equivalente en agua alcanza entre 13 millones de $\mathrm{m}^{3}$ y 19 millones de $\mathrm{m}^{3}$. Entre 1988 y 1990 se comenzó con una primera etapa de depositación sobre este glaciar rocoso, emplazando $14 \mathrm{Mt}$ en dos depósitos de $40 \mathrm{~m}$ de espesor, o sea de un espesor similar al promedio del glaciar rocoso (Contreras e Illanes, 1992; Valenzuela, 2004). Estos depósitos se sitúan entre los $3.960 \mathrm{~m}$ y $4.000 \mathrm{~m}$ de altura, casi $500 \mathrm{~m}$ sobre las instalaciones productivas de la mina.
Como consecuencia de la depositación se observó un fuerte aumento en la velocidad de desplazamiento superficial del glaciar rocoso Infiernillo. La velocidad aumentó de entre 0,3 $\mathrm{m}$ por año y $1,9 \mathrm{~m}$ por año en estado natural hasta un máximo de $25 \mathrm{~m}$ por año después de la depositación, alcanzándose los valores más altos de desplazamiento 4 a 5 meses después del inicio (Contreras e Illanes, 1992; Valenzuela, 2004). Debido a este aceleramiento inesperado se abandonaron los planes iniciales de depositar $30 \mathrm{Mt}$ adicionales en el glaciar rocoso (Valenzuela, 2004). Más de 10 años después de la depositación todavía se observan tasas elevadas entre $1 \mathrm{~m}$ y $13 \mathrm{~m}$ por año, con un promedio de 5,8 m por año (Valenzuela, 2004). Valenzuela (2004) también menciona en forma explícita la posibilidad de una falla de tales depósitos sobre glaciares rocosos.

En el caso de División Andina se comenzó en la década de 1980 a construir depósitos de roca de baja ley sobre glaciares rocosos (Morales-Carvajal, 2001). El efecto de sobrecarga aumentó la velocidad de desplazamiento hasta $30 \mathrm{~m}$ por año o $35 \mathrm{~m}$ por año, causando inestabilidades en los bancos superiores del rajo y afectando la operación de la mina (Apablaza et al., 2001). Esta situación hizo necesario el monitoreo permanente

Figura $\mathrm{N}^{\circ} 3$

Glaciar rocoso cerro Negro en los años 1982 y 2007

a)

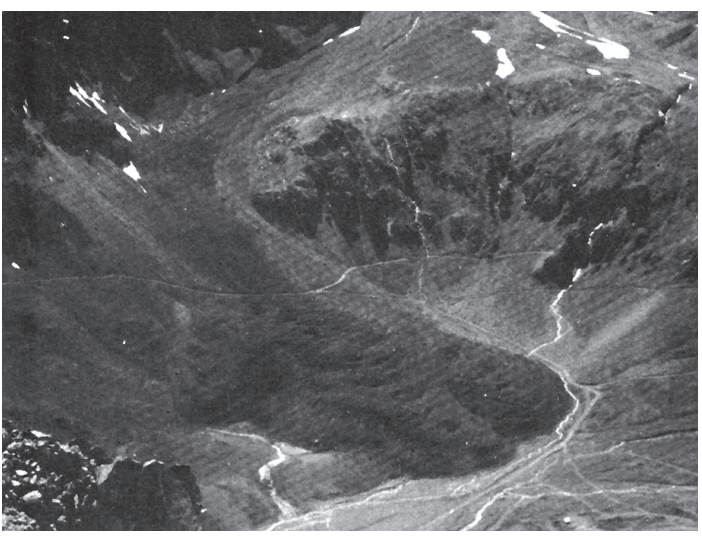

b)

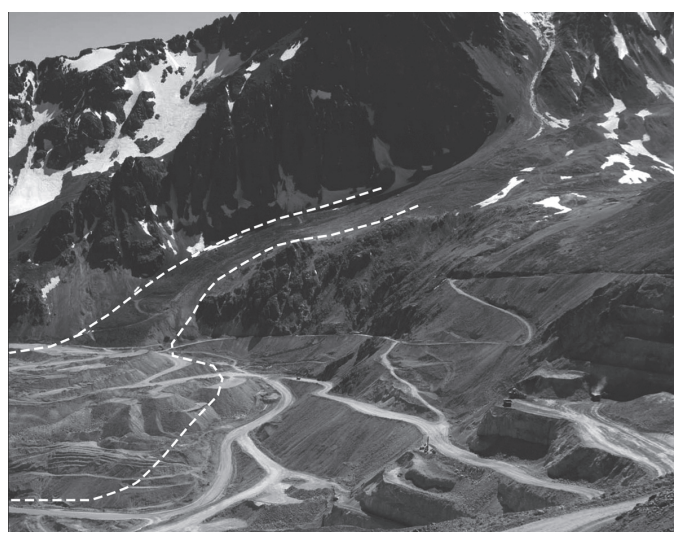

Fuente: Barsch, 1988 y fotografía base del archivo Codelco. 
de las laderas para detectar zonas potencialmente inestables antes de que se genere una falla (Apablaza et al., 2001). Para poder controlar el desplazamiento y la estabilidad del depósito sobre el glaciar rocoso, se removió material de este para alcanzar una tasa de desplazamiento anual de $20 \mathrm{~m}$, una meta que se logró hasta el año 2000-2001 (Apablaza et al., 2001). Sin embargo, el avance continuo del glaciar rocoso requiere que su material sea removido de manera regular (Apablaza et al., 2001).

La situación geomorfológica de una eventual falla de un glaciar rocoso cargado con roca estéril en Los Bronces o División Andina es similar a la situación del derrumbe y flujo detrítico catastrófico del río Colorado en 1987. Durante este derrumbe aproximadamente $30 \mathrm{Mt}$ de roca y probablemente hielo se desprendieron desde una ladera ubicada entre los 4.000 m.s.n.m. y 4.500 m.s.n.m. (depósito Infiernillo: 4.000 m.s.n.m., sobrecarga de $14 \mathrm{Mt}$ ). Las masas se desplazaron primero en forma de derrumbe y después como flujo de barro $40 \mathrm{~km}$ valle abajo, causando la pérdida de 43 vidas (Casassa y Marangunic, 1993).

A modo de comparación, las masas de un posible colapso del depósito de lastre y glaciar rocoso Infiernillo podrían mezclarse con agua y sedimentos finos del tranque de relave de Los Bronces, generando de esta forma un flujo detrítico a lo largo del río San FranciscoMapocho, con efectos destructivos de carácter catastrófico para la ciudad de Santiago, a $40 \mathrm{~km}$ de distancia. Al respecto Valenzuela (2004) enfatiza que uno de los aspectos que no ha recibido la atención suficiente es la posibilidad de la falla de una vertiente seguida por un flujo detrítico que podría desplazarse una distancia considerable río abajo del depósito, lo que podría representar un serio peligro para la población (Valenzuela, 2004).

\section{Los Pelambres, Región de Coquimbo}

Minera Los Pelambres $\left(31^{\circ} 43^{\prime} \mathrm{S}, 70^{\circ}\right.$ $29^{\prime}$ W), propiedad de Antofagasta Minerals $(60 \%)$ y un consorcio japonés, se localiza en la cuenca superior del río Choapa. La producción del año 2005 fue de 332 kt de cobre (Minergía, 2006b). La mina comenzó sus operaciones en el año 1992 con una producción alrededor de $20 \mathrm{kt}$ de cobre al año. En el año 1997 se presentó un proyecto de expansión al SEIA, calificado favorablemente, realizando el cambio de minería subterránea a extracción a rajo abierto (Geotécnica, 1997). En los años 2002 y 2004 se iniciaron nuevas etapas de ampliación con el fin de mantener constante la producción de cobre fino a pesar de la caída de las leyes del yacimiento (Editec, 2006).

Azócar y Brenning (2008) examinaron las intervenciones de Los Pelambres en glaciares rocosos sobre la base de fotografías aéreas e imágenes satelitales tomadas entre 1997 y 2006, identificándose 15 glaciares rocosos en los alrededores de la mina, además de la intervención por faenas mineras de varios glaciares rocosos ubicados al noreste y este del rajo de la mina. Cuatro glaciares rocosos localizados en la parte noroeste han desaparecido casi completamente por la depositación de lastre sobre estos (el área de glaciares rocosos afectados es $0,11 \mathrm{~km}^{2}$ ). Por otro lado, al este del rajo han sido intervenidos dos glaciares rocosos por la construcción de caminos, equivalentes a 0,14km² (Azócar y Brenning, 2008). Las intervenciones sobre glaciares rocosos se realizaron durante los años 2004-2005, en forma paralela al avance de los depósitos de estériles en el área (Azócar y Brenning, 2008). Estas actividades de remoción y depositación no fueron anunciadas en los estudios ambientales presentados a la autoridad entre los años 1997 y 2004. Sin embargo, se conocía de su existencia desde el año 1998 (Geoestudios, 1998). Minera Los Pelambres niega la existencia de glaciares rocosos en el área (La Nación, 2008), aunque el análisis de fotografías aéreas de la zona no deja lugar a dudas.

\section{Pascua-Lama, Región de Atacama}

Pascua-Lama $\left(29^{\circ} 11^{\prime} \mathrm{S}, 70^{\circ} 3^{\prime} \mathrm{W}\right)$ es el primer proyecto minero binacional (chilenoargentino) perteneciente a la empresa canadiense Barrick Gold Corporation. Las reservas probadas de este proyecto de minería a rajo abierto son de 17 millones de onzas de oro y 635 millones de onzas de plata, y se estima una vida útil de 20 años con una producción anual de al menos 750 mil onzas de oro y 30 millones de onzas de plata (Editec, 2006). El ElA está aprobado y el proyecto se 
encuentra en etapa de construcción por el lado chileno.

Pascua-Lama inició su proceso de aprobación ambiental el año 2001, aprobándose en una primera instancia la remoción de los campos de nieve perenne Toro 1 (área actual $\left.0,07 \mathrm{~km}^{2}\right)$, Toro $2\left(0,07 \mathrm{~km}^{2}\right)$ y Esperanza, $\left(0,01 \mathrm{~km}^{2}\right)$ (Nicholson y Marín, 2008). Estos muchas veces fueron interpretados en el discurso público como glaciares, pero se consideran aquí como campos de nieve por ser de tamaño menor a 0,1 km² (Haeberli, 2000). El proyecto se postergó por razones económicas y políticas hasta el año 2004, año en que se presenta un nuevo EIA que enfrentó la fuerte oposición de grupos ambientalistas, agricultores y gobierno (Kronenberg, 2009). El proyecto finalmente fue aprobado en el 2006, prohibiéndose la remoción y alteración de los campos de nieve anteriormente mencionados.

Un hecho poco conocido públicamente pero presente en el EIA aprobado en 2006, es que el desarrollo del proyecto implica la destrucción de un pequeño glaciar rocoso denominado Estrecho-B $\left(0,034 \mathrm{~km}^{2}\right.$, equivalente en agua a entre 0,24 millones de $\mathrm{m}^{3} \mathrm{y}$ 0,37 millones de $\mathrm{m}^{3}$ ), el que será cubierto por el avance del botadero de estériles Nevada Norte cinco años después del inicio de la explotación (Golder Associates, 2006). A modo de comparación, el equivalente en agua afectada en Codelco División Andina es casi 100 veces mayor que en Pascua-Lama.

\section{Minas y Proyectos con Impactos Menores}

Otras minas y proyectos en diferentes fases de desarrollo han causado solo menores impactos en glaciares rocosos debido a la construcción de caminos de exploración (Figura $N^{\circ} 3$, Cuadro $N^{\circ} 2$ ). Entre ellos están Codelco División El Teniente (cinco glaciares rocosos intervenidos), una de las minas subterráneas de cobre más grandes del mundo, el proyecto Cerro Casale (un glaciar rocoso intervenido), en los Andes áridos con ocurrencia aislada de glaciares rocosos, el proyecto Catedral (dos glaciares rocosos intervenidos), y la mina Pimentón (al menos cuatro glaciares rocosos intervenidos). Esta última $\left(32^{\circ}\right.$ $29^{\prime}$ S, 70 $12^{\prime} \mathrm{W}$; South American Gold and Copper Company), una pequeña mina subte- rránea que explota vetas de alta ley de cobre y oro, podría producir un impacto mayor en el caso de una eventual explotación a rajo abierto de un pórfido más significativo existente debajo de glaciares rocosos.

\section{Proyección al futuro}

Los impactos mineros en glaciares rocosos son difíciles de predecir dado que algunos de los proyectos se encuentran en fases muy tempranas de prospección o en fase de evaluación ambiental o económica. Es posible identificar, sin embargo, de manera preliminar, algunos proyectos cuyo desarrollo merece mayor atención en los próximos años por encontrarse en zonas muy cercanas a glaciares rocosos, o por ya haber causado menores intervenciones en glaciares rocosos tales como la construcción de caminos y la ejecución de sondajes. También se evalúan en forma preliminar los impactos de los proyectos en el territorio argentino.

\section{Proyectos en Chile}

Los potenciales impactos futuros asociados a posibles expansiones de Codelco División Andina (prospecto El Americano) y mina Pimentón como asimismo el posible comienzo de las operaciones en Pascua-Lama y Catedral ya se mencionaron previamente. Las minas Pimentón, El Teniente (ambas subterráneas en la actualidad) y Cerro Casale que hasta ahora solo han causado impactos menores en glaciares rocosos podrían implicar intervenciones mayores si se realizan expansiones futuras o si el modo de explotación cambia de subterráneo a superficial como ha ocurrido en Los Pelambres. Varias otras prospecciones, en cambio, constituyen proyectos menos avanzados que, por su ubicación, potencialmente afectarán glaciares rocosos (prospectos Tordillo, Novicio, West Wall, Las Lagunillas y Amos Andrés; mina Flor de los Andes) (Figura $\mathrm{N}^{\circ} 3$ ).

\section{Proyectos en Argentina}

Argentina ha visto un gran auge en su producción minera desde la década del 1990. Llegó a ser el tercer productor de oro en las Américas, y se espera un aumento de la producción de metales del 40\% en 2009 (Kroll, 2009). Tan solo en 2008, las perforaciones 
de exploración en Argentina superaron los 660 km, 3 veces más que en 2003 (Argentina Mining, 2009). Gran parte de los actuales proyectos en fase de operación, desarrollo o exploración se concentran en las zonas cordilleranas de San Juan y provincias vecinas en la extensión oriental de los cinturones cupríferos y auríferos de El Teniente-Río BlanCo, El Indio y Maricunga. Los antecedentes existentes sobre las minas y los proyectos de exploración dan una primera impresión de los posibles impactos futuros que el desarroIlo minero en Argentina puede causar si tales intervenciones se concretan. Ya se observan impactos menores en glaciares rocosos debido a la construcción de caminos en los proyectos El Pachón, Los Azules, y probablemente El Altar, donde, sin embargo, las imágenes disponibles fueron de calidad limitada. Otros proyectos están asociados con posibles intervenciones futuras en glaciares rocosos debido a su ubicación, pero la mayoría de ellos se encuentra en fases tempranas de exploración. Estos son el proyecto ya avanzado de Lama (parte argentina de Pascua-Lama) y la mina Veladero, además de los prospectos (de norte a sur) Agua Rica, la zona Vicuña (prospectos Vicuña, Las Flechas, Josemaría, Los Helados, Sillimanita, Batidero), Mogote, Del Carmen, Río Cenicero, Rincones de Araya, Los Bagres y Amos Andrés (Figura No 3).

\section{Proyecto en Perú}

En Perú, hasta la fecha se observan intervenciones en glaciares rocosos en solo un caso (Shila-Paula, dos glaciares rocosos intervenidos por caminos, área total de $0,2 \mathrm{~km}^{2}$ ), mientras es probable que el futuro desarrollo minero del país implique más intervenciones.

\section{Consideraciones finales}

El estudio de los aspectos políticos-legales y los fundamentos científicos de intervenciones antrópicas en la criósfera de alta montaña es un tema de interés científico bastante reciente. Hasta ahora los impactos mineros sobre la criósfera han recibido poca atención (Brenning, 2008; Kronenberg, 2009). En los Alpes europeos, la discusión está principalmente vinculada a los derechos de propiedad privada en glaciares, el esquí y la construcción de andariveles sobre glaciares, y los residuos de munición de ejercicios mi- litares (Haimayer, 1989; Wiesflecker, 2003; Hasslacher, 2005; Bütler, 2007). Además de la legislación a nivel nacional o provincial/ cantonal, en los Alpes, una serie de acuerdos internacionales como la Convención Alpina y sus protocolos y, en el caso de los países miembros de la Unión Europea, la Directiva Marco del Agua, brindan cierta protección a los glaciares (Bütler, 2007). Una protección explícita podría implementarse a través de un Protocolo del Agua propuesto en el marco de la Convención Alpina (Bütler, 2007). Si bien en el contexto alpino varios de los aspectos legales de los glaciares propiamente dichos también se aplicarían a los glaciares rocosos al tratarse de recursos hídricos y elementos del paisaje, no se ha realizado un análisis específico relacionado a estos últimos, por lo cual es difícil comparar las situaciones chilena y alpina.

En cuanto a la legislación relacionada a los glaciares en Chile, la modificación reciente al SEIA y la implementación de la PNG, con las limitaciones analizadas en este artículo, son los primeros reglamentos que explícitamente establecen su protección como objetivo de la política del Estado chileno. La modificación al SEIA permitirá reconocer más tempranamente algunos de los proyectos mineros que puedan afectar glaciares rocosos, al menos cuando los inventarios necesarios se hayan recopilado. Sin embargo, el ejemplo de la aprobación de la remoción de glaciares rocosos declarada en el EIA de la expansión de División Andina en 2001 sugiere que el SEIA por sí solo no es un instrumento que puede garantizar la conservación de glaciares rocosos (Brenning, 2008). El ejemplo de la prohibición de remover glaciares en el proyecto Pascua-Lama en 2006, sin embargo, subraya la importancia de la participación ciudadana en la protección de sus recursos (Brenning, 2008; Kronenberg, 2009). Aunque la cantidad de hielo de glaciares rocosos afectada por actividades mineras es menor a la pérdida total de hielo glacial en Chile central atribuida al cambio climático (Bown et al., 2008; Le Quesne, 2009), la sociedad puede más fácilmente contrarrestar los efectos adversos de la minería (Kronenberg, 2009).

El presente trabajo ha identificado 26 faenas mineras en Chile, Argentina y Perú que han intervenido glaciares rocosos o podrían 
intervenirlos en el futuro. Hasta la fecha, el área afectada de glaciares rocosos en Chile es de aproximadamente $3,3 \mathrm{~km}^{2}$, la mayor parte concentrada en Codelco División Andina y Los Bronces (Brenning, 2008). A modo de comparación, el glaciar Juncal Norte $(7,6$ $\mathrm{km}^{2}$ ), uno de los glaciares más grandes cercanos a la ciudad de Santiago, perdió 1,5 $\mathrm{km}^{2}$ de su área en un período de 51 años, entre 1955 y 2006 (Bown et al., 2008). El equivalente en agua correspondiente al área intervenida de glaciares rocosos es del orden de 30 millones de $\mathrm{m}^{3}$. Para poner este número en contexto, el embalse La Laguna en la cuenca del río Elqui almacena en promedio alrededor de 23 millones de $\mathrm{m}^{3}$ de agua y el embalse El Yeso (cuenca del río Maipo), alrededor de 180 millones de $\mathrm{m}^{3}$.

Un asunto que hasta ahora ha recibido muy poca atención en Chile son los peligros antropogénicos relacionados a la minería en permafrost de montaña. En particular, se debe considerar la posibilidad de inestabilidades en depósitos de lastre que causen flujos detríticos secundarios (Valenzuela, 2004). Tales inestabilidades pueden ocurrir tanto en el corto plazo como dentro de décadas o siglos, dependiendo de las medidas de cierre de minas tomadas y el desarrollo de la estructura interna del permafrost de glaciares rocosos. Por analogía al evento natural de 1987 (Casassa y Marangunic, 1993) se debe considerar la posibilidad que tal flujo detrítico afectara zonas pobladas, incluso el borde urbano de Santiago.

Más allá de los impactos de la minería de alta cordillera en glaciares rocosos, la ubicación de un gran número de proyectos mineros en las cabeceras de las principales cuencas hidrográficas de los Andes semiáridos de Chile y Argentina implica que, en el largo plazo (segunda mitad de este siglo), muchas de estas cuencas van a estar sujetas a los efectos geoquímicos (ARD) y geomorfológicos (movimientos en masa y erosión de depósitos y rajos), los que afectarán en una forma u otra a ríos y zonas pobladas aguas abajo con procesos como filtración y dispersión de aguas contaminadas, flujos detríticos y aluviones causados por movimientos en masa en la zona de minas cerradas o abandonadas, entre otros (Hartman et al., 2005; Palmer et al., 2010). Los ejemplos de tales efectos de largo plazo abundan en los países con una historia minera más larga. El costo asociado a estos fenómenos quedará con la sociedad y el Estado. Para evitar potenciales daños en la mayoría de las cuencas de los Andes semiáridos, en propia opinión, sería necesario elaborar ahora planes y escenarios que permitan conservar aquellas cuencas de importancia estratégica para las poblaciones y los ecosistemas.

La minería altoandina, explotando principalmente yacimientos de baja ley en minas a rajo abierto de gran escala, es un fenómeno similar a la controvertida "decapitación de cerros" (mountaintop removal, MTR) en la minería de carbón del este de Estados Unidos (Szwilski et al., 2001; Palmer et al., 2010). Las críticas al MTR enfatizan principalmente el impacto paisajístico, la destrucción de los bosques y suelos que los sustentan y la depositación de material en las cabeceras de los valles afectando la calidad del agua y la biodiversidad aguas abajo (Palmer et al., 2010). También destaca el hecho que estos proyectos pueden afectar varias cuencas hidrográficas a la vez, multiplicando los impactos ambientales. Aspectos similares se pueden mencionar en el caso de la minería en los Andes secos, agregando que el agua es un recurso mucho más escaso en esta zona, y los ambientes de alta montaña son geomorfológicamente más dinámicos que los Apalaches. Por otro lado, el Tratado de Integración y Complementación Minera entre Chile y Argentina, firmado el año 1997, que tiene por objetivo facilitar la inversión minera en zonas fronterizas, permitirá el desarrollo de varios proyectos en las cabeceras hidrográficas limítrofes de ambos países, en su mayoría caracterizadas por la presencia de glaciares blancosy rocosos.

Finalmente, uno de los pasos futuros en el ámbito científico y político debe ser la consideración de los servicios ambientales y valores no económicos relacionados con los glaciares rocosos. En este trabajo se presentó una clasificación general de tales servicios y valores basada en Kronenberg (2009). Esta clasificación, junto con la tipología de intervenciones en glaciares rocosos y sus posibles efectos, puede guiar estudios concretos en glaciares rocosos andinos y contribuir a una mejor valoración de estos fenómenos del 
permafrost de alta montaña en el contexto de la implementación de la nueva Política Nacional de Glaciares de Chile e iniciativas similares en Argentina.

\section{Agradecimientos}

Esta investigación fue financiada por el Department of Foreign Affairs and International Trade (DFAIT) de Canadá y el Natural Sciences and Engineering Research Council of Canada (NSERC). Los autores agradecen a la Sra. M. Rankl (Universidad de Erlangen, Alemania) su amable cooperación en la búsqueda de información.

\section{Referencias bibliográficas}

APABLAZA, R.; FARÍAS, E.; MORALES, R.; DÍAZ, J. \& KARZULOVIC, A. The Sur Sur mine of Codelco's Andina division. In: HUSTRULID, W. A.; MCCARTER, M. K. \& VAN ZYL, D. J. A. Slope stability in surface mining. Colorado: Society for Mining Metallurgy and Exploration, 2001, p. 171176.

ÁREA MINERA. Codelco tiene nueva reserva de cobre en Andina y mejora sus reservas en 20\%. Revista Área Minera, 2009. Disponible en Internet: http://www.aminera. com/noticias-generales/1-latest-news/16986codelco-tienen-nueva-reserva-de-cobre-enandina-y-mejora-sus-reservas-en-20-.pdf

ARGENTINA MINING. Mining in Argentina. Mendoza: Argentina Mining, 2009. Disponible en Internet: http://www. argentinamining.com/en/mineria-enargentina/

ARENSON, L.; HOELZLE, M. \& SPRINGMAN, S. Borehole deformation measurements and internal structure of some rock glaciers in Switzerland. Permafrost and Periglacial Processes, 2002, No 13, p. 117 135.

AZÓCAR, G. F. y BRENNING, A. Intervenciones en glaciares rocosos en Minera Los Pelambres, Región de Coquimbo, Chile. Waterloo: University of Waterloo, informe técnico, 2008.
AZÓCAR, G. F. \& BRENNING, A. Hydrological and geomorphological significance of rock glaciers in the dry Andes, Chile $\left(27^{\circ}-33^{\circ} \mathrm{S}\right)$. Permafrost and Periglacial Processes, 2010, № 21, p. 42-53.

BARSCH, D. Rockglaciers. In: CLARK, M. J. (ed.). Advances in Periglacial Geomorphology. Chichester: John Wiley \& Sons, 1988, p. 69-90.

BARSCH, D. Rockglaciers. Berlin: Springer, 1996.

BÓRQUEZ, R.; LARRAÍN, S.; POLANCO, R. y URQUIDI, J.C. Glaciares chilenos: reservas estratégicas de agua dulce para la sociedad, los ecosistemas y la economía. Santiago: LOM Ediciones, 2006.

BOWN, F.; RIVERA, A. \& ACUÑA, C. Recent glacier variations at the Aconcagua basin, central Chilean Andes. Annals of Glaciology, 2008, No 48, p. 43-48.

BRENNING, A. Geomorphological, hydrological and climatic significance of rock glaciers in the Andes of Central Chile (33$\left.35^{\circ} \mathrm{S}\right)$. Permafrost and Periglacial Processes, 2005, No 16, p. 231-240.

BRENNING, A. The impact of mining on rock glaciers and glaciers: examples from Central Chile. In: ORLOVE, B. S.; WIEGANDT, E. \& LUCKMAN, B. Darkening peaks: glacier retreat, science, and society. Berkeley: University of California Press, 2008, p. 196-205.

BURGER, K. C.; DEGENHARDT, J. J. \& GIARDINO, J. R. Engineering geomorphology of rock glaciers. Geomorphology, 1999, N ${ }^{\circ}$ 31, p. 93-132.

BUSINESS NEWS AMERICAS. Miners concerned over glacier protection bill. Business News Americas, 2007. Disponible en Internet: http://www.bnamericas.com/ news/mining/Miners_concerned_over_ glacier_protection_bill

BÜTLER, M. Glaciers as objects of law and international treaties. In: PSENNER, R. \& LACKNER, R. Alpine space-man \& 
environment, vol. 3, The water balance of the Alps, What do we need to protect the water resources of the Alps? Proceedings of the Conference held at Innsbruck University, 2829 September 2006. Innsbruck: Universidad de Innsbruck, 2007, p. 19-31.

CASASSA, G. \& MARANGUNIC, C. The 1987 Rio Colorado rockslide and debris flow, Central Andes, Chile. Bulletin of the Association of Engineering Geologists, 1993, No 30 , p. 321-330.

CODELCO. Memoria Anual Codelco 2007. Santiago: Codelco, 2008.

COMISIÓN NACIONAL DEL MEDIO AMBIENTE (CONAMA). Política para la Protección y Conservación de Glaciares. Santiago: CONAMA, 2009.

CONTRERAS, A. e ILLANES, J. L. Depósito de lastre glaciar Infiernillo Sur, mina Los Bronces. En: Santiago, Minería en altura, 43a Convención del Instituto de Ingenieros de Minas de Chile, La Serena, 1992.

CUFF, D. J. Resources. In: GOUDIE, A. S. Encyclopedia of Global Change. Oxford: Oxford University Press, 2008. Disponible en Internet: http://www.oxford-globalchange. com/entry?entry=t178.e0382

EDITEC. Compendio de la minería chilena. Santiago: Editec, 2006.

EL MOSTRADOR. Ley de protección de glaciares en el freezer. El Mostrador. Publicación periódica de Santiago, Chile, 3 de diciembre de 2008. Disponible en Internet: http://www.elmostrador.cl/index. php?/noticias/imprimir/ley_de_protecci/

GEOESTUDIOS. Estudio de glaciares de roca en el área de mina Los Pelambres. Informe técnico \# 376 para Bechtel Chile Ltda. Santiago: Geoestudios Ltda., 1998.

GEOTÉCNICA. Estudio de impacto ambiental: proyecto de expansión minera Los Pelambres $85.000 \mathrm{tpd}$, resumen ejecutivo. Santiago: Geotécnica Consultores, 1997.
GREBENETS, V. I.; KERIMOV, A. G. \& BAKCHEEV, D. S. Dangerous movements of technogenic rock glaciers, Norilsk, Russia. In: MARINOS, P. G.; KOUKIS, G. C.; TSIAMBAOS, G. C. \& STOURNARAS, G. C. Engineering geology and the environment. Rotterdam: Balkema, 1997, p. 689-692.

GOLDER ASSOCIATES. Estudio de impacto ambiental Modificaciones proyecto Pascua-Lama. Santiago: Golder Associates, 2006.

HAEBERLI, W. Modern research perspectives relating to permafrost creep and rock glaciers: a discussion. Permafrost and Periglacial Processes, 2000, No 11, p. 290293.

HAIMAYER, P. Glacier-skiing areas in Austria - a socio-political perspective. Mountain Research and Development, 1989, No 9, p. 51-58.

HARTMAN, K. J.; KALLER, M. D.; HOWELL, J. W. \& SWEKA, J. A. How much do valley fills influence headwater streams? Hydrobiologia, 2005, No 532, p. 91-102.

HASSLACHER, P. Gletscherschutz -ein wichtiger Baustein der alpinen Raumordnung. In: Bedrohte Alpengletscher. Innsbruck: Oesterreichischer Alpenverein, Alpine Raumordnung, No 27, 2005, p. 7-15.

HOELZLE, M.; WAGNER, S.; KÄ̈̈B, A. \& VONDER MÜHLL, D. Surface movement and internal deformation of ice-rock mixtures within rock glaciers at PontresinaSchafberg, Upper Engadin, Switzerland. In: LEWKOWICZ, A. G. \& ALLARD, M. Proceedings of the Seventh International Conference on Permafrost, Yellowknife, Canada. Quebec City: Centre d'Études Nordiques, Nordicana, No 57, 1998, p. 465471.

HOLLESEN, J.; ELBERLING, B. \& HANSEN, B. U. Modelling subsurface temperatures in a heat producing coal waste rock pile, Svalbard $\left(78^{\circ} \mathrm{N}\right)$. Cold Regions Science and Technology, 2009, No 58, p. 68-76. 
KÄ̈̈B, A.; HUGGEL, C.; FISCHER, L.; GEUX, S.; PAUL, F.; ROER, I.; SALZMANN, N.; SCHLAEFLI, S.; SCHMUTZ, K.; SCHNEIDER, D.; STROZZI, T. \& WEIDMANN, $Y$. Remote sensing of glacier -and permafrostrelated hazards in high mountains: an overview. Natural Hazards and Earth System Sciences, 2005, № 5, p. 527-554.

KÄ̈̈B, A.; FRAUENFELDER, R. \& ROER, I. On the response of rockglacier creep to surface temperature increase. Global and Planetary Change, 2007, No 56, p. 172-187.

KROLL, C. Argentina will launch four mining projects in 2009. Engineering \& Mining Journal, 2009, No 210, p. 40-42.

KRONENBERG, J. Global warming, glaciers and gold mining. In: Ljubljana, Transformation, Innovation and Adaptation for Sustainability, 8th International Conference of the European Society for Ecological Economics, 2009.

LA NACIÓN. Los Pelambres en la mira por glaciares rocosos. Diario La Nación. Publicación periódica de Santiago, Chile, 16 de octubre de 2008.

LE QUESNE, C.; ACUÑA, C.; BONINSEGNA, J. A.; RIVERA, A. \& BARICHIVICH, J. Long-term glacier variations in the Central Andes of Argentina and Chile, inferred from historical records and tree-ring reconstructed precipitation. Palaeogeography Palaeoclimatology Palaeoecology, 2009, N ${ }^{\circ}$ 281, p. 334-344.

LLIBOUTRY, L. Phénomènes cryoniveaux dans les Andes de Santiago (Chili). Biuletyn Peryglacjalny, 1961, No 10, p. 209-224.

MINERGÍA. Con la colaboración de las mineras, el Senado busca proteger y valorar los glaciares. Minergía, 2006a, № 68, p. 38-39.

MINERGía. Minera Los Pelambres. Minergía, 2006b, № 66, p. 8-18.

MORALES-CARVAJAL, R. F. Manejo de morrenas en mina Sur-Sur. Memoria título profesional ingeniero en minas. Santiago: Departamento de Ingeniería de Minas, Universidad de Chile, Chile, 2001.
NICHOLSON, L. y MARÍN J. Inventario de los cuerpos de hielo y glaciares rocosos en la cuenca superior del río Huasco. Centro de Estudios Avanzados en Zonas Áridas (CEAZA). Informe No 2008-10. La Serena: Centro de Estudios Avanzados en Zonas Áridas, 2008.

PALMER, M. A.; BERNHARDT, E. S.; SCHLESINGER, W. H.; ESHLEMAN, K. N.; FOUFOULA-GEORGIOU, E.; HENDRYX, M. S.; LEMLY, A. D.; LIKENS, G. E.; LOUCKS, O. L.; POWER, M. E.; WHITE, P. S. \& WILCOCK, $\mathrm{P}$. R. Mountaintop mining consequences. Science, 2010, No 327, p. 148-149.

PARKS CANADA. Kluane National Park and Reserve of Canada -Summary of Trails and Routes: Rock Glacier Trail. Quebec: Parks Canada, 2008. Disponible en Internet: http://www.pc.gc.ca/eng/pn-np/yt/kluane/ activ/activ1/activ1b/v.aspx

ROER, I.; K $\ddot{A} \ddot{B} B, A$. \& DIKAU, R. Rockglacier kinematics derived from small-scale aerial photography and digital airborne pushbroom imagery. Zeitschrift für Geomorphologie, 2005, № 49, p. 73-87.

SZWILSKI, T. B.; DULIN, B. E. \& HOOPER, J. W. An innovative approach to managing the environmental impacts of mountaintop coal mining in West Virginia. International Journal of Mining, Reclamation and Environment, 2001, № 15, p. 73-85.

TROMBOTTO, D.; BUK, E. \& HERNÁNDEZ, J. Rock glaciers in the Southern Central Andes (approx. $33^{\circ}-34^{\circ} \mathrm{S}$ ), Cordillera Frontal, Mendoza, Argentina. Bamberger Geographische Schriften, 1999, $N^{\circ} 19$, p. 145-173.

VALENZUELA, L. Stability issues in natural and man made slopes in mining. In: LACERDA, W. A.; EHRLICH, M.; FONTOURA, S. A. B. \& SAYÃO, A. S. F. Landslides: evaluation and stabilization. Leiden: Balkema, 2004, p. 467-473.

WIESFLECKER, H. Gletscherschutz in der Alpenregion. Tesis de doctorado. Innsbruck: Universidad de Innsbruck, Austria, 2003. 